\title{
Endoscopic ultrasound-guided fine needle aspiration for the diagnosis of bowel endometriosis: a case report
}

\author{
Takao Miwa ${ }^{1}$, Takuji Iwashita ${ }^{2}$, Masashi Aiba ${ }^{1}$, Takahiro Kochi' ${ }^{1}$, Katsuhisa Toda ${ }^{1}$, Noriaki \\ Nakamura $^{1}$, Naoki Katsumura ${ }^{1}$, Masahito Shimizu ${ }^{2}$
}

${ }^{1}$ Department of Gastroenterology, Chuno Kosei Hospital, ${ }^{2}$ Department of Gastroenterology, Gifu University Graduate School of Medicine, Gifu, Japan

\begin{abstract}
Bowel endometriosis is a condition caused by endometrial glands and stroma infiltrating the bowel wall and reaching the subserous fat tissue or the adjacent subserous plexus. A 42-year-old woman with changes in stool habits, nausea, and stomach aches experienced complete obstruction in the rectum. Endoscopic ultrasound demonstrated a low echoic lesion outside the rectal mucosa and endoscopic ultrasound-guided fine needle aspiration confirmed the diagnosis of bowel endometriosis. The clinical characteristics of bowel endometriosis are unspecific and this condition is sometimes misdiagnosed as a malignant tumor, irritable bowel syndrome, or any other colorectal disorder. Our aim is to show that endoscopic ultrasound-guided fine needle aspiration might be helpful for bowel endometriosis diagnosis and exclusion of other malignant disease.

Keywords: bowel endometriosis; bowel obstruction; endoscopic ultrasound-guided fine needle aspiration; ileus
\end{abstract}

\section{Introduction}

Bowel endometriosis is a condition in which endometrial glands infiltrates the bowel wall, mostly in the sigmoid colon and rectum ( $\geq 90 \%)$; it is reported in $3.8-37 \%$ of patients with endometriosis [1,2]. Due to nonspecific clinical characteristics, bowel endometriosis is occasionally misdiagnosed as a malignant tumor, irritable bowel syndrome, or other colorectal disorders $[3,4]$. Since a

Received 03.05.2018 Accepted 14.06.2019

Med Ultrason

2020, Vol. 22, No 2, 243-246

Corresponding author: Takuji Iwashita, MD, PhD

Department of Gastroenterology,

Gifu University Graduate School of Medicine

1-1 Yanagido, Gifu, 501-1194, Japan

Phone: +81-58-230-6308

Fax: +81-58-230-6310

E-mail: takuji@w7.dion.ne.jp differential diagnosis of bowel endometriosis from other malignancies with imaging studies is difficult, histological confirmation using the laparoscopic approach is usually required if bowel endometriosis is suspected by clinical findings [5]. Endoscopic ultrasound-guided fine needle aspiration (EUS-FNA) might be an option to obtain specimens from endometrial glands and stroma infiltrating the bowel wall; however, its efficacy has been barely evaluated.

Here, we report a case of bowel endometriosis in which EUS-FNA was useful to establish a diagnosis and determine a management plan without laparoscopic or surgical procedures.

\section{Case report}

A 42-year-old woman had previously underwent total hysterectomy and bilateral salpingectomy for uterine myoma including ovarian chocolate cyst removal. Three 
months previously, she was referred to our clinic owing to nausea, constipation, and lower abdominal cramping pain. Blood examination showed slightly elevated serum cancer antigen 125 level $(44.4 \mathrm{U} / \mathrm{mL})$. Imaging work-up revealed niveau formation in the ascending colon on abdominal radiography (fig 1a) and enhanced thickening of the rectal wall on contrast enhanced computed tomography (CT) (fig 1b). These findings suggested the presence of colonic ileus caused by rectal cancer or post-surgical stricture from the hysterectomy. The colonic ileus was successfully managed with conservative treatment, including nasal ileus tube placement. After resolution of the ileus, colonoscopy was performed, which revealed complete obstruction in the upper rectum (fig 1c, 1d). Endoscopic biopsy did not show any obvious abnormal findings, although it was difficult to rule out malignancies. Subsequently, EUS (GF-UC240P; Olympus, Tokyo, Japan) was performed, which demonstrated a $27.3 \times 18.8 \mathrm{~mm}$ low echoic lesion below the rectal mucosa and submucosa, infiltrating the rectal muscularis propria (fig 2a). EUS-FNA was performed using a 22-gauge needle (Acquire; Boston Scientific, MA, USA) (fig 2b). Pathological evaluation revealed endometrial glands and stroma scattered in smooth muscle tissue; additional immunohistochemical stains revealed cluster differentiation 10 in the surrounding stroma and estrogen receptor in the endometrial glands (fig 3). The lesion was finally diagnosed as bowel endometriosis and managed by endoscopic balloon dilatation and administration of dienogest as hormone therapy, which improved her symptoms. Fol-
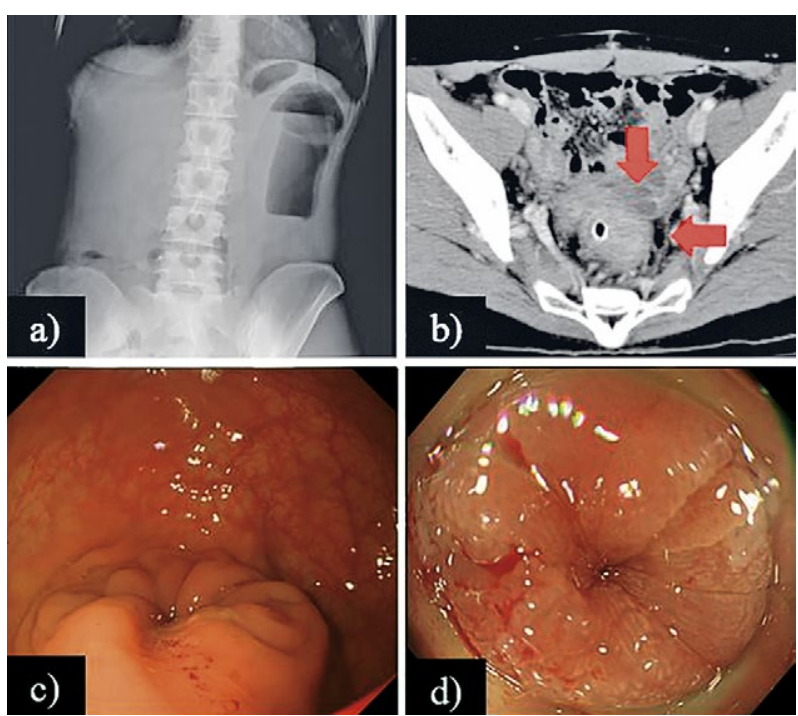

Fig 1. a) Abdominal radiographs showing niveau formation in the ascending colon; b) computed tomography with contrast showing enhanced thickening of the rectum wall (arrow); c) and d) colonoscopy showing complete obstruction in the rectum. low-up CT performed 14 months later also showed improvement of rectal wall thickness.

\section{Discussions}

EUS is an established imaging modality to examine the lower gastrointestinal tract and its high resolution enables recognition of the layer structure of the rectal wall. EUS has been reported to have high accuracy in $\mathrm{T}$ staging, especially in rectal cancer [6]. Imaging features of bowel endometriosis on EUS were reported as a hypoechoic lesion with an irregular and unclear margin [7]. Another advantage of EUS in bowel endometriosis is the evaluation of bowel infiltration as $\mathrm{T}$ staging for rectal cancer, which is important to determine management methods, either laparoscopy or laparotomy [8], and whether bowel resection is necessary [9]. However, despite precise evaluation with EUS, pathological evalu-

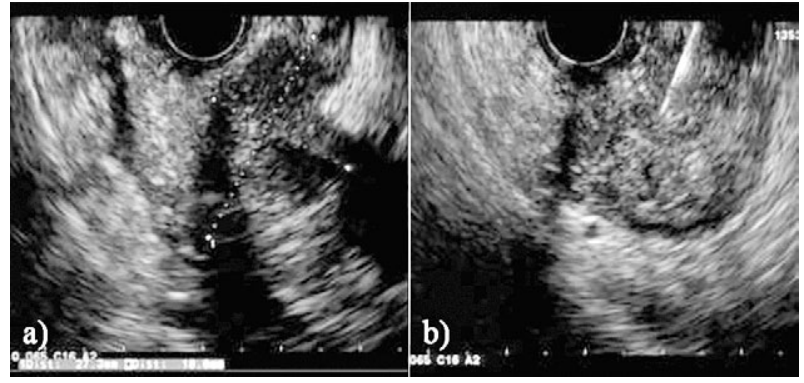

Fig 2. a) Endoscopic ultrasound demonstrated a $27.3 \times 18.8 \mathrm{~mm}$ low echoic lesion infiltrating the rectal muscularis propria; b) endoscopic ultrasound-guided fine needle aspiration was performed using a 22-gauge needle.

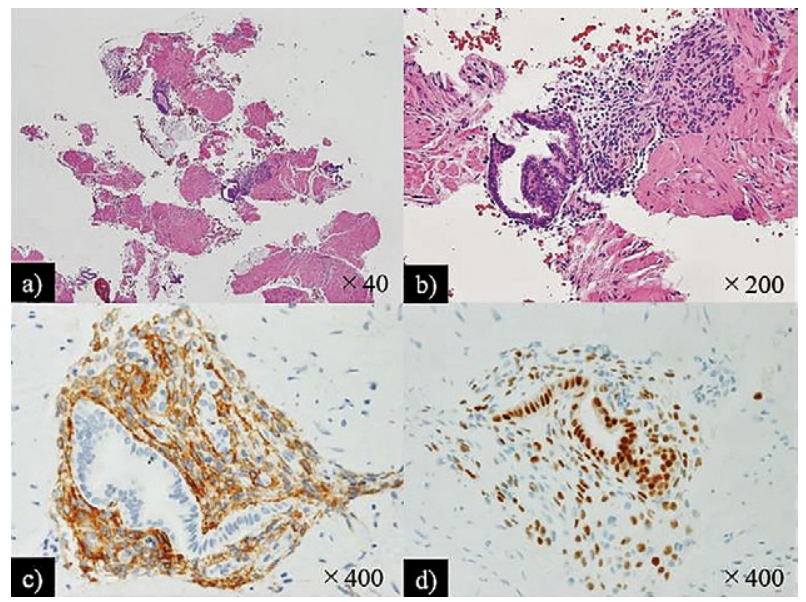

Fig 3. a) and b) Pathological evaluation revealed endometrial glands and stroma scattered in the smooth muscle tissue; c) cluster of differentiation 10 was positive in the surrounding stroma; d) the estrogen receptor was positive in the endometrial glands. 
ation is vital to establish a definite diagnosis of bowel endometriosis.

Another advantage of EUS is its ability to perform pathological sampling using FNA under EUS guidance, which was first reported in 1992 [10]. EUS-FNA has been reported as a reliable method to obtain pathological specimens of pelvic lesions located in the intraluminal or around the lower intestine $[11,12]$. However, the efficacy of EUS-FNA for endometriosis has been barely evaluated and was highlighted only in a few case reports or case series $[7,13,14]$ (Table I.). Together with the findings of these reports and our case, the pooled sensitivity of EUS-FNA for bowel endometriosis was 70\% (7/10) $[7,13,14]$. Surgical exploration or operation could be prevented in 3 of 7 patients diagnosed using EUS-FNA; conservative treatment was administered instead. Thus, EUS-FNA could help to obtain a pathological diagnosis of bowel endometriosis and change the management plan by preventing surgical exploration. As for safety, compared with upper intestinal EUS-FNA, lower gastrointestinal EUS-FNA has been reported to be associated with higher rates of adverse events (20.5\%), although most adverse events were mild [15]. Therefore, the indications of EUS-FNA for pelvic lesions need to be carefully considered; however, given their low invasive nature, we believe that EUS-FNA should be performed if there is any possibility that the FNA diagnosis could change the management plan, such as that for colon cancer versus bowel endometriosis.

In our case, malignant diseases or post-surgical effects from the patient's hysterectomy were initially suspected as the cause of rectal obstruction based on blood test and imaging evaluations. EUS revealed hypoechoic tumors at the obstruction site and following FNA, successfully provided a pathological diagnosis of bowel endometriosis without any adverse events. EUS-FNA was useful to prevent unnecessary surgery and changed the management method to hormonal therapy with endoscopic balloon dilation.

In conclusion, EUS is a useful examination to evaluate the cause of obstruction in the rectum, and EUS-FNA should be considered if a pathological diagnosis cannot be established using regular endoscopic biopsy. EUSFNA is also useful to diagnose bowel endometriosis around the rectum.

Acknowledgement: Authors would like to acknowledge the assistance of Dr. Naoki Ito for his clinical support and Dr. Nami Asano and Dr. Tamotsu Takeuchi for their academic support.

\section{References}

1. Remorgida V, Ferrero S, Fulcheri E, Ragni N, Martin DC. Bowel endometriosis: presentation, diagnosis, and treatment. Obstet Gynecol Surv 2007;62:461-470.

2. Campagnacci R, Perretta S, Guerrieri M, et al. Laparoscopic colorectal resection for endometriosis. Surg Endosc 2005; 19:662-664.

3. Seaman HE, Ballard KD, Wright JT, de Vries CS. Endometriosis and its coexistence with irritable bowel syndrome and pelvic inflammatory disease: findings from a national case-control study--Part 2. BJOG 2008;115:1392-1396.

4. Wolthuis AM, Meuleman C, Tomassetti C, D'Hooghe T, de Buck van Overstraeten A, D'Hoore A. Bowel endometriosis: colorectal surgeon's perspective in a multidisciplinary surgical team. World J Gastroenterol 2014;20:1561615623.

5. Dunselman GA, Vermeulen N, Becker C, et al. ESHRE guideline: management of women with endometriosis. Hum Reprod 2014;29:400-412.

6. Jurgensen C, Teubner A, Habeck JO, Diener F, Scherubl H, Stolzel U. Staging of rectal cancer by EUS: depth of

Table I. Characteristics of reported patients with bowel endometriosis who underwent EUS-FNA

\begin{tabular}{|c|c|c|c|c|c|c|}
\hline No. & Age & EUS findings & EUS-FNA diagnosis & $\begin{array}{l}\text { Pathological } \\
\text { diagnosis }\end{array}$ & Treatment & Reference \\
\hline 1 & 41 & Hypoechoic, regular border lesion & Endometriosis & EUS-FNA & Surgical & {$[13]$} \\
\hline 2 & 37 & Hypoechoic, regular border lesion & Endometriosis & EUS-FNA & Conservative & [13] \\
\hline 3 & 50 & Hypoechoic, irregular border lesion & Endometriosis & EUS-FNA & Conservative & {$[13]$} \\
\hline 4 & 36 & Heterogenous, irregular border lesion & Endometriosis & EUS-FNA & NA & [7] \\
\hline 5 & 44 & Heterogenous, irregular border lesion & Inconclusive & None & Conservative & [7] \\
\hline 6 & 42 & Hypoechoic lesion & Inconclusive & Surgical exploration & Conservative & {$[7]$} \\
\hline 7 & 53 & Hypoechoic lesion & Inconclusive & Surgical exploration & Surgical & {$[7]$} \\
\hline 8 & 26 & Hypoechoic lesion & $\begin{array}{l}\text { Strongly suggestive } \\
\text { of endometriosis }\end{array}$ & EUS-FNA & NA & {$[7]$} \\
\hline 9 & NA & NA & Endometriosis & EUS-FNA & NA & {$[14]$} \\
\hline 10 & 42 & Hypoechoic, irregular border lesion & Endometriosis & EUS-FNA & Conservative & Our case \\
\hline
\end{tabular}

EUS-FNA, endoscopic ultrasound-guided fine needle aspiration; NA, not available. 
infiltration in T3 cancers is important. Gastrointest Endosc 2011;73:325-328.

7. Pishvaian AC, Ahlawat SK, Garvin D, Haddad NG. Role of EUS and EUS-guided FNA in the diagnosis of symptomatic rectosigmoid endometriosis. Gastrointest Endosc 2006;63:331-335.

8. Chapron C, Dumontier I, Dousset B, et al. Results and role of rectal endoscopic ultrasonography for patients with deep pelvic endometriosis. Hum Reprod 1998;13:2266-2270.

9. Desplats V, Vitte RL, du Cheyron J, Roseau G, Fauconnier A, Moryoussef F. Preoperative rectosigmoid endoscopic ultrasonography predicts the need for bowel resection in endometriosis. World J Gastroenterol 2019;25:696706.

10. Vilmann P, Jacobsen GK, Henriksen FW, Hancke S. Endoscopic ultrasonography with guided fine needle aspiration biopsy in pancreatic disease. Gastrointest Endosc 1992;38:172-173.
11. Mohamadnejad M, Al-Haddad MA, Sherman S, McHenry L, Leblanc JK, DeWitt J. Utility of EUS-guided biopsy of extramural pelvic masses. Gastrointest Endosc 2012;75:146-151.

12. Sasaki Y, Niwa Y, Hirooka Y, et al. The use of endoscopic ultrasound-guided fine-needle aspiration for investigation of submucosal and extrinsic masses of the colon and rectum. Endoscopy 2005;37:154-160.

13. Hara K, Yamao K, Ohashi K, et al. Endoscopic ultrasonography and endoscopic ultrasound-guided fine-needle aspiration biopsy for the diagnosis of lower digestive tract disease. Endoscopy 2003;35:966-969.

14. Leyden J, Winter DC, Clarke E, O'Keane C. Endoscopic ultrasound and EUS-guided FNA in the diagnosis of rectal endometriosis. Ir Med J 2009;102:301.

15. Levy MJ, Abu Dayyeh BK, Fujii LL, et al. Prospective evaluation of adverse events following lower gastrointestinal tract EUS FNA. Am J Gastroenterol 2014;109:676-685. 